\title{
Advances in patch clamp technique: towards higher quality and quantity
}

\author{
Markéta Bébarová \\ Department of Physiology, Faculty of Medicine, Masaryk University, Kamenice 5, 62500 Brno - Bohunice, Czech Republic
}

\begin{abstract}
The patch clamp technique, developed in late 1970s, started a new period of experimental cardiac electrophysiology enabling measurement of ionic currents on isolated cardiomyocytes down to the level of single channels. Since that time, the technique has been substantially improved by development of several upgraded modifications providing so far unavailable data (e.g. action potential clamp, dynamic clamp, high-resolution scanning patch clamp), or facilitating the patch clamp technique by increasing its efficiency (planar patch clamp, automated patch clamp). The current review summarizes the leading new patch clamp based techniques used in cardiac cellular electrophysiology, their principles and prominent related papers.
\end{abstract}

Key words: Action potential clamp - Dynamic clamp - Cell-type transforming clamp - Highresolution scanning patch clamp - Automated patch clamp

\section{Introduction}

Based on his experiments which considerably contributed to the formulation of theory of electrical excitation, Luigi Galvani anticipated the existence of ionic channels already at the end of $18^{\text {th }}$ century (Piccolino 1998). Nevertheless, the assumed structure of plasmalemma was supplemented with associated proteins including ionic channels as late as in the 1930s (Danielli and Davson 1935). Direct data proving their existence were still missing.

In 1939, Hodgkin and Huxley began to work together. They measured both the membrane voltage and currents in the squid giant axons using longitudinal metal electrodes. Unfortunately, their work was interrupted by the World War II. In 1952, they published a series of five papers describing the results of their experiments. In the last paper (Huxley and Hodgkin 1952), they united all the acquired data and transformed them into a mathematical model which resulted in determination of the laws that govern the movement of ions in a nerve cell during action potential (AP).

In the meantime, to acquire direct data proving the existence of ionic channels, a special method was sought capable to get a sufficiently tight connection with a small region of the cellular membrane providing the possibility

Correspondence to: Markéta Bébarová, Department of Physiology, Faculty of Medicine, Masaryk University, Kamenice 5, 62500 Brno

- Bohunice, Czech Republic

E-mail: bebarova.lfmu@centrum.cz to record electrical processes with a low level of undesirable noise. In 1949, measurement of the membrane voltage using penetrating glass microelectrodes filled with $3 \mathrm{M}$ potassium chloride was first introduced (Ling and Gerard 1949). Later modification of this technique which used apposing glass microelectrodes resulted in development of the patch clamp technique. It was first used by Neher and Sakmann (1976) to analyse ionic currents through single acetylcholineactivated membrane channels in the frog skeletal muscle. Many improvements of the patch clamp technique have been introduced since that time. In this review, a summary of the most important upgraded modifications of the patch clamp technique and their application in the cardiac cellular electrophysiology is presented.

\section{Conventional patch clamp technique}

The principle of the patch clamp technique has been most recently described by e.g. Ogden and Stanfield (1994); Karmažínová and Lacinová (2010). Shortly, it is based on an electrical isolation of a patch of membrane from the external solution which makes it possible to measure the current flowing into the patch. Fire-polished glass microelectrodes filled with an electrolyte solution are gently pressed against the membrane and a light suction is then applied to get the high-resistance seal (usually called gigaseal because the resistance should exceed $10 \mathrm{G} \Omega$ to satisfactorily reduce noise). The measurements may be done either on the 
whole-cell or single channel level (cell-attached, inside- or outside-out), depending on the final configuration of the microelectrode and cell membrane, and on composition of the solution inside the microelectrode (the pipette solution) and outside the cell (the bath solution; e.g. Ogden and Stanfield 1994).

The whole-cell mode is the most often used mode of the patch clamp technique. In the current clamp mode, recorded are either spontaneous APs in spontaneously active cells (e.g. sinus node cells) or APs in spontaneously inactive cells (atrial and ventricular myocytes) stimulated by short supra-threshold current pulses. If voltage pulses (most often rectangular) are applied, ionic membrane current may be measured (the voltage clamp mode). To separate the particular ionic currents, voltage pulses are used of various duration and amplitude (based on knowledge of time- and voltage-dependent characteristics of the currents), as well as specific inhibitors.

\section{Examples of upgraded modifications of patch clamp technique}

\section{AP clamp}

In contrast to conventional patch clamp where rectangular (or less often ramp) voltage pulses are used to elicit ionic currents, a native AP waveform is used for stimulation of the measured cell in the course of AP clamp experiment. This method, which shifts the patch clamp measurements closer to physiological conditions, was first used in isolated cardiomyocytes by Doerr et al. $(1989,1990)$. The key advantage of AP clamp is the possibility to analyse particular ionic currents and related subcellular processes during the course of natural AP waveform and explore their contribution to its formation. Despite providing interesting data, it is used rather exceptionally. The reason might be that the conventional voltage clamp measurements offer precise voltage and time characteristics of the ionic channel function which can be more easily compared among various laboratories and used for adjustment of mathematical models of cardiac cells.

Regarding some of the recently published papers based on AP clamp data, Stengl et al. (2010) proved a linkage between the diminished L-type calcium current and AP shortening as observed in isolated ventricular cardiomyocytes and right ventricular trabeculae, respectively, of a clinically relevant porcine model of hyperdynamic septic shock. Cooper et al. (2010) found that $\mathrm{Ca}^{2+}$ release from the sarcoplasmic reticulum is significantly decreased when the cell is stimulated with AP waveform characteristic for the cardiac failure (an absence of AP notch). This behaviour indicates an impaired excitation-contraction coupling. The currently available possibilities of use of AP clamp to test the selectivity of compounds affecting cardiac ionic channels have been recently reviewed by Szentandrássy et al. (2011).

Several modifications of AP clamp technique have been introduced. Banyasz et al. (2011) developed the so-called AP clamp sequential dissection technique. It is based on recording the steady-state AP in the current clamp mode which is subsequently used to stimulate the same cell in the voltage clamp mode. If the properties of the cell did not change since the time of AP recording, no current is measured in the control conditions (baseline current; Fig. 1A). Unstable cells with non-zero baseline current were not included in the study. After adding a specific inhibitor, the compensatory current can be recorded. The inhibited current can be obtained by subtracting the compensatory current from the baseline current. If another specific inhibitor is added (Fig. 1B), the compensatory current is formed by both inhibited currents. The second current can be then separated by subtracting the second compensatory current from the first one. Other specific inhibitors can be then sequentially added to separate further currents. Thus, this technique enables to concurrently record several ionic currents in a single cardiomyocyte (Fig. 1C). These authors revealed a large variability of ionic currents among isolated guinea-pig ventricular cardiomyocytes and also observed that ionic currents in a cardiomyocyte are coordinated.

\section{Dynamic clamp}

The dynamic clamp technique (reviewed by Wilders 2006) is based on conventional current clamp. It is characterized by stimulation of the measured cell with the real-time recorded or simulated current which is a function of the freerunning APs. This method was first introduced by S. Scott in 1979 who electrically connected two independent groups of isolated cardiomyocytes free of physical contact. It enabled their interaction and essentially functioned as virtual gap junctions. The dynamic clamp was then further worked up and applied also in other cell types, e.g. in endocrine cells or neurons. Nowadays, it is even more often used in neurophysiology than in cardiac electrophysiology, e.g. in studies focused on the synaptic activity (Economo et al. 2010). Three basic configurations of the dynamic clamp technique are used in the cardiac cellular electrophysiology: the coupling clamp, model clamp and dynamic AP clamp (Fig. 2).

\section{Coupling clamp}

The coupling clamp configuration (Fig. 2A) simulates the above mentioned electrical connection (coupling) of cells. The membrane potentials of both cells $\left(V_{m, 1}\right.$ and $\left.V_{m, 2}\right)$ are recorded in the current clamp mode. The recorded values of 
$V_{m, 1}$ and $V_{m, 2}$ are sampled into a personal computer (PC) which computes, based on the difference between $V_{m, 1}$ and $V_{m, 2}$, the coupling current $I_{c}$ "flowing" from myocyte 1 to 2 (i.e. the difference of their total ionic membrane currents) in the real-time. Then, PC discharges the command potentials $V_{c m d, 1}$ and $V_{c m d, 2}$ to the respective amplifiers which generate the required current pulse contributing to the total membrane current and, thereby, affecting the membrane potential. If the appropriate $I_{c}$ is applied, $V_{m, 1}$ and $V_{m, 2}$ can be synchronized. The coupling clamp has been used to study e.g. electrotonic modulation of the pacemaker activity of the sinoatrial node by the atrial muscle (Watanabe et al. 1995), or the interaction between Purkinje cells and ventricular cardiomyocytes (Huelsing et al. 1998). In some of these studies (e.g. Watanabe et al. 1995), one of the cells is replaced by a model of cardiomyocyte.

\section{Model clamp}

In case of the model clamp configuration (Fig. 2B), the dynamic clamp measurement is performed only in one cell whose $V_{m}$ is continuously sampled to PC computing the $V_{m}$-dependent current $I_{x}$. This current is enriched by simulated additional conductance. Eventually, the corresponding command potential $V_{c m d}$ is amplified and injected into the myocyte. This method was used to study, among others, the impact of depolarized, inexcitable cardiomyocytes in the ischemic tissue on the neighbouring cells (the so-called injury current; Verkerk et al. 2000), or consequences of artificially implemented specific ionic current in cells where it is not naturally present (e.g. transient outward potassium current $I_{t o}$ in guinea-pig or canine endocardial ventricular myocytes - Dong et al. 2006). Even the whole model cell may be interconnected with the measured cell (as mentioned above) or the measured

Figure 1. Action potential clamp sequential dissection technique. When the measured cell is stimulated with its own steady-state action potential, no current is recorded (baseline). After adding of chromanol-293B, a specific inhibitor of slow delayed rectifier potassium current $I_{K s}$, the compensatory current will appear. $I_{K s}$ can be obtained by subtracting the compensatory current from the baseline (A). After adding of E4031 (inhibitor of fast delayed rectifier potassium current $I_{K r}$ ), the compensatory current is formed by both $I_{K s}$ and $I_{K r} . I_{K r}$ can be then separated by subtracting the second compensatory current from the first one (B). Using additional specific inhibitors sequentially (e.g. nisoldipine to inhibit calcium current $I_{C a}$ and $\mathrm{Ba}^{2+}$ to inhibit inward rectifier potassium current $I_{K 1}$ ), this technique makes it possible to concurrently record several ionic currents in a single cardiomyocyte $(\mathbf{C}) . V_{m}$, membrane potential; $I_{m}$, membrane current. Modified with permission from Banyasz et al. (2011).

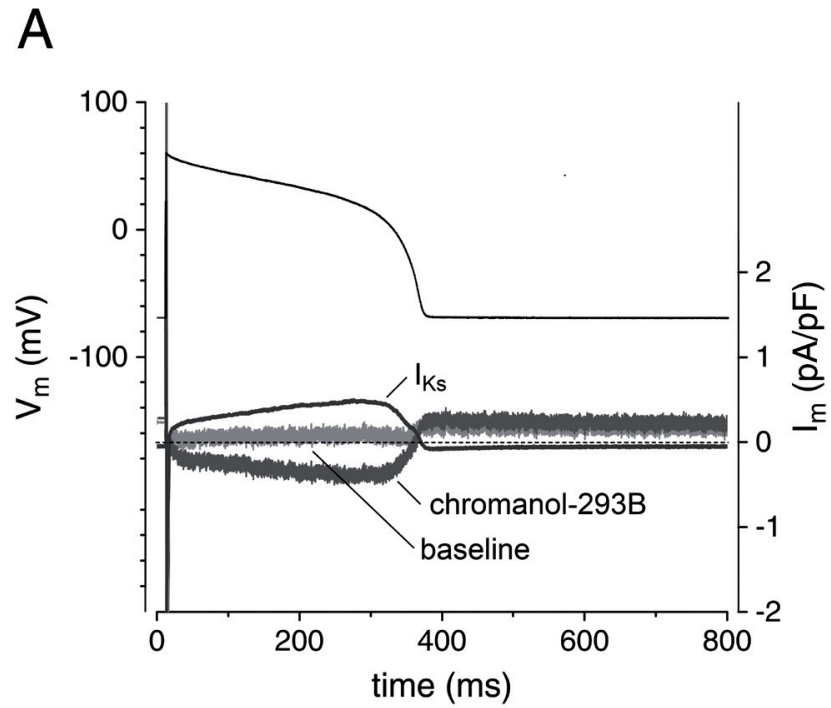

B

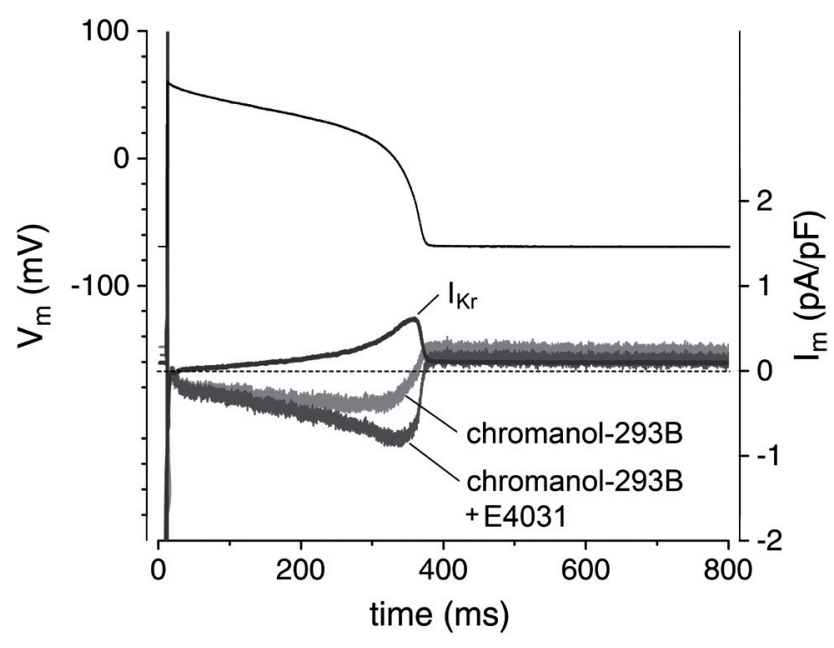

C

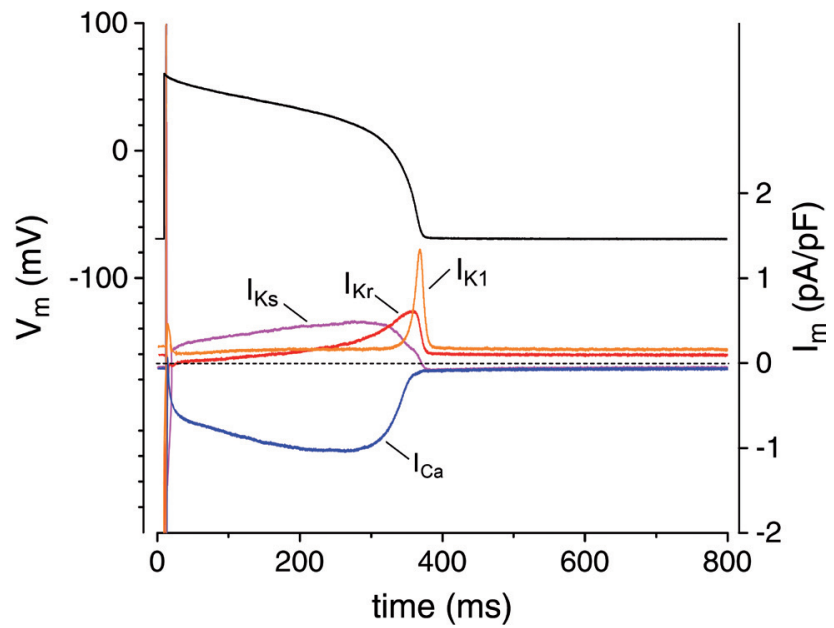



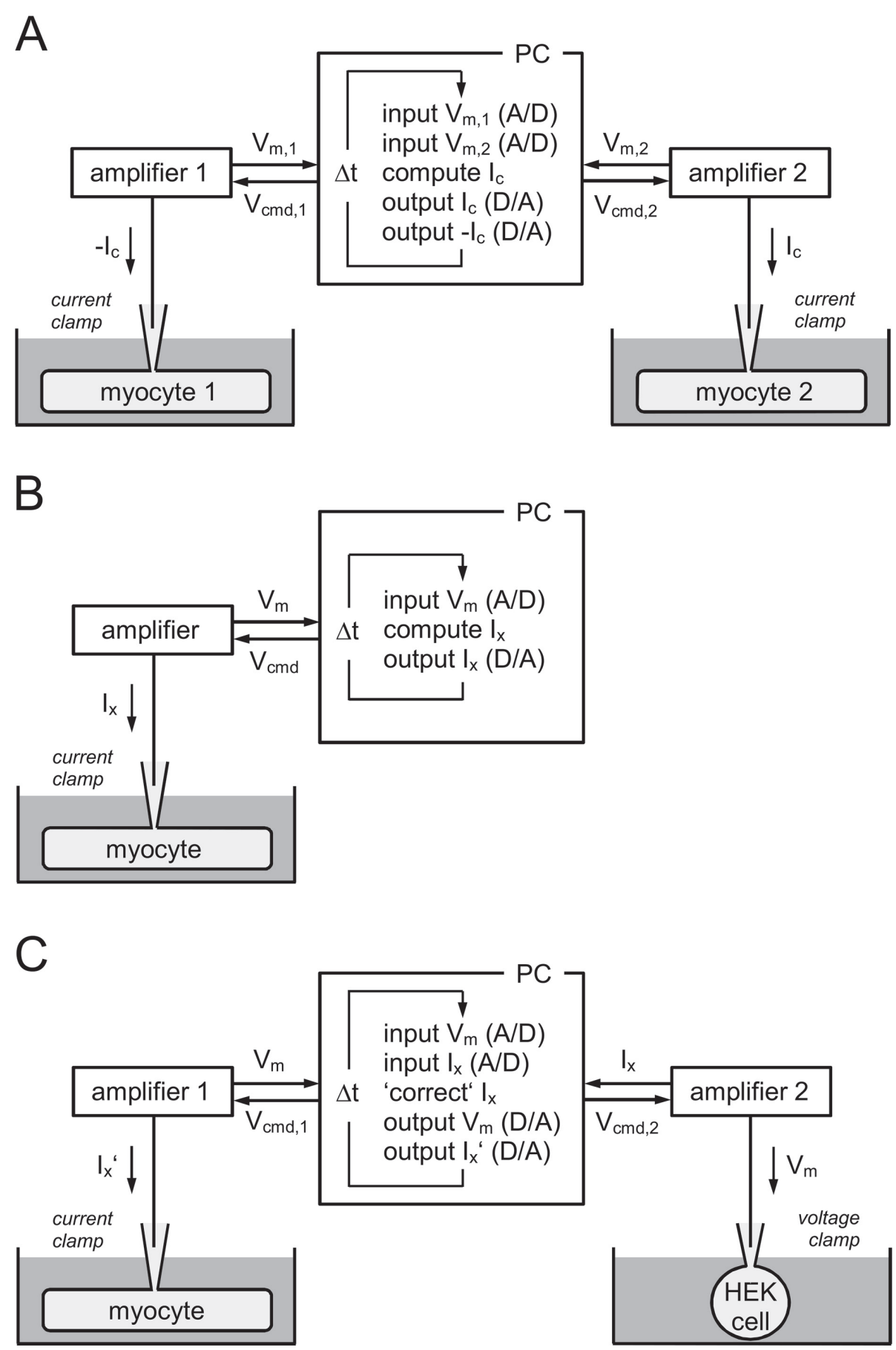

Figure 2. Three configurations of dynamic clamp usually used in cardiac cellular electrophysiology. A. Coupling clamp. Membrane potentials of both cells $\left(V_{m, 1}\right.$ and $\left.V_{m, 2}\right)$ are recorded and sampled into a personal computer (PC) which computes, based on the difference between $V_{m, 1}$ and $V_{m, 2}$, the coupling current $I_{c}$ (i.e. the difference of total ionic membrane currents of both myocytes) in the real-time. Then, PC discharges the command potentials $V_{c m d, 1}$ and $V_{c m d, 2}$ to the respective amplifiers which generate the required current pulse to synchronize $V_{m, 1}$ and $V_{m, 2}$. B. Model clamp. The dynamic clamp measurement is performed only in one cell whose $V_{m}$ is continuously sampled to PC computing the $V_{m}$-dependent current $I_{x}$. This current is enriched by simulated additional conductance. Eventually, the corresponding command potential $V_{c m d}$ is amplified and injected into the myocyte. C. Dynamic action potential clamp. The analysed cardiac ionic current is specifically inhibited and replaced by an ionic current conducted through the required ionic channels, either wild-type or mutant, expressed in a cell line, e.g. in the human embryonic kidney (HEK) 293 cells. The cell line cell is stimulated with AP waveform continuously recorded from the cardiomyocyte. Modified with permission from Wilders (2006). 
cell may be placed into a virtual network of myocytes (e.g. Wang et al. 2000).

\section{Dynamic AP clamp}

Berecki et al. (2005) described another configuration of the dynamic clamp which combines AP clamp and dynamic clamp techniques and is called the dynamic AP clamp (Fig. 2C). The analysed cardiac ionic current is specifically inhibited (or abolished if a model of cardiomyocyte is used) and replaced by an ionic current measured in a cell line, e.g. in the human embryonic kidney (HEK) 293 cells in Berecki et al. (2005). On the surface of these cells, only the required ionic channel is expressed, either wild-type or mutant. The cell line cell is stimulated with AP waveform continuously recorded from the cardiomyocyte (or generated by the model). Berecki et al. (2005) used this technique to readily and unambiguously determine the effect of $h E R G$ mutation R56Q (identified in a patient with long QT syndrome) on AP configuration in particular layers of ventricular myocardium. Electrophysiological properties of R56Q- $h E R G$ mutation found out with the conventional whole-cell patch clamp technique showed controversial changes of AP duration. The observed faster deactivation was supposed to result in AP prolongation whereas the contemporary present faster activation should theoretically cause AP shortening. The dynamic AP clamp proved that this mutation leads to AP prolongation. Berecki et al. (2006) used the same technique also in the subsequent study focused on an analogical analysis of two sodium channel mutations identified in patients with long QT syndrome. Possible applications of the dynamic AP clamp in the studies analysing the consequences of cardiac ionic channel mutations related to inherited arrhythmias were resumed in 2007 (Berecki et al. 2007). Another scientific group has recently applied this method in the same way to reveal the effects of a sodium channel mutation identified in a patient with Brugada syndrome (Marangoni et al. 2011).

\section{Benefits and limitations of dynamic clamp}

The described modifications of the dynamic clamp and their utilization in cardiac cellular electrophysiology clearly indicate that this method offers direct responses to many issues related to the basic cellular mechanisms of AP formation, propagation and synchronisation in both healthy and diseased myocardium. On the other hand, the dynamic clamp, as any other experimental technique, has its limitations. First, it is a quite challenging technique requiring simultaneous measurement from two cells (except for the model clamp). In case of the model clamp, the used mathematical description of ionic current has to be accurate indeed to provide reliable results. An impor- tant limitation is that the injected current enters the cell through the glass microelectrode rather than through the real ionic channels which makes impossible to study the signal conduction effects of the current, and may result in differences in ions carrying the current and local changes of ionic concentrations. Other limitations are related to the technical demands, e.g. keeping latency between the acquiring voltage and applying the current based on that voltage as short as possible, or scaling the current measured in the expression system before its injection to the myocyte in a proper way (dynamic AP clamp). Hence, much of expertise is required to perform experiments with the dynamic clamp technique, however, the invested effort may be rewarded by interesting and relevant data.

\section{Cell-type transforming clamp}

Recently, Ahrens-Nicklas and Christini (2009) have introduced a new method which, similarly to the dynamic AP clamp, is based on both AP clamp and dynamic clamp techniques. It is called the cell-type transforming clamp and should be able to suppress the interspecies differences of AP configuration in the real time. As shown in Figure $3 \mathrm{~A}$, the membrane voltage of the target cell (the one whose characteristics are required to be changed; here the mouse cardiomyocyte) is measured and put into to the targetcancelling model and recipient model (the model of the desired cell type, e.g. human cardiomyocyte). Each model cell current $\left(I_{\text {cancel }}\right.$ and $I_{\text {recip }}$, respectively) is calculated and the difference current $\left(I_{\text {diff }}\right)$ is then obtained. The current compensating the seal leak $\left(I_{\text {seal }}\right)$ and stimulus current $\left(I_{s-}\right.$ tim) are subsequently added to $I_{\text {diff }}$ to produce the injected current $\left(I_{\text {inj }}\right)$ which is injected into the target cell. It results in a change of AP configuration in the mouse cardiomyocyte (Fig. 3B) to that typical for the human one including identical dynamics of changes of AP waveform (Fig. 3C). Considering a notably higher availability of mouse cardiomyocytes, this technique might provide data relevant for human cardiac electrophysiology in a much easier and likely also cheaper way.

However, several limitations have to be taken into account when using this technique. The eligibility of compensation of differences in particular components of the total membrane ionic current between the measured (target) and required (model) cell type notably depends on the quality of the used model. Even if the model is superior, it is, however, always created to fit the data under specific experimental conditions. Thus, running the model under conditions far from those used to make it may induce considerable inaccuracies in the cell dynamics. Errors in the circuit (caused by mismatch between the target cell and the target-cancelling model, or mismeasurement of the target cell capacitance) unwillingly modifying the obtained results may also ap- 
A

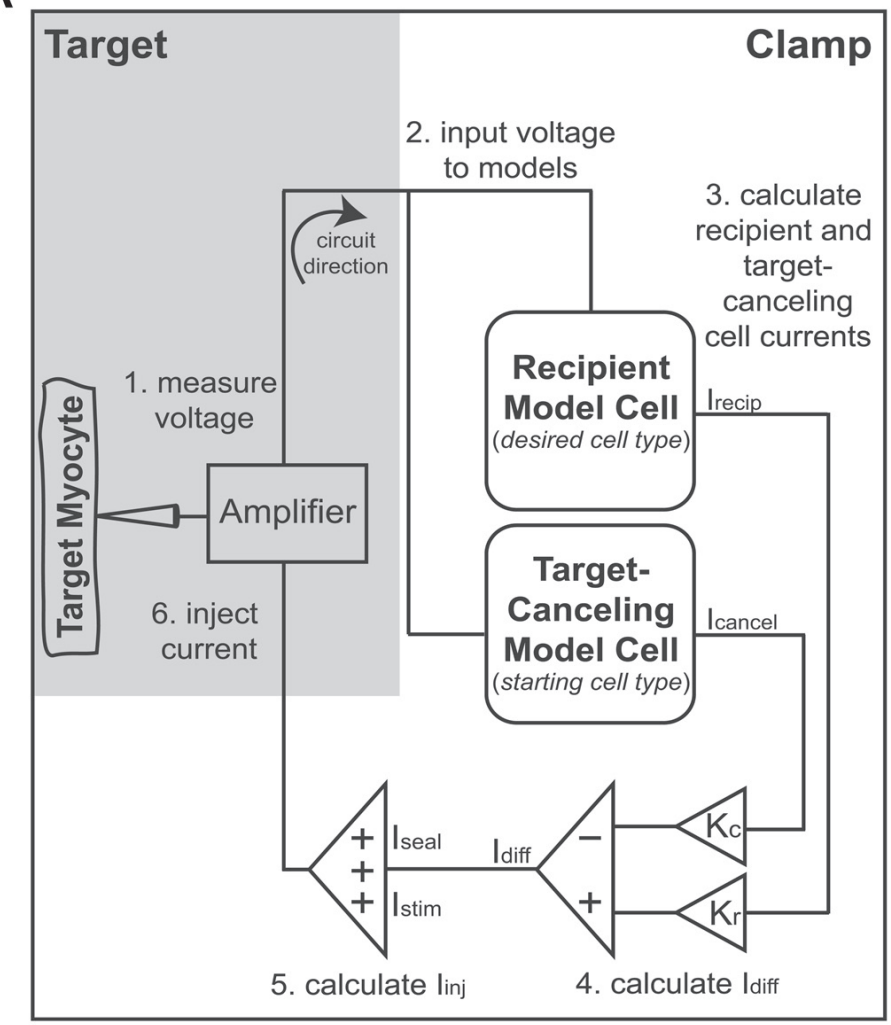

B

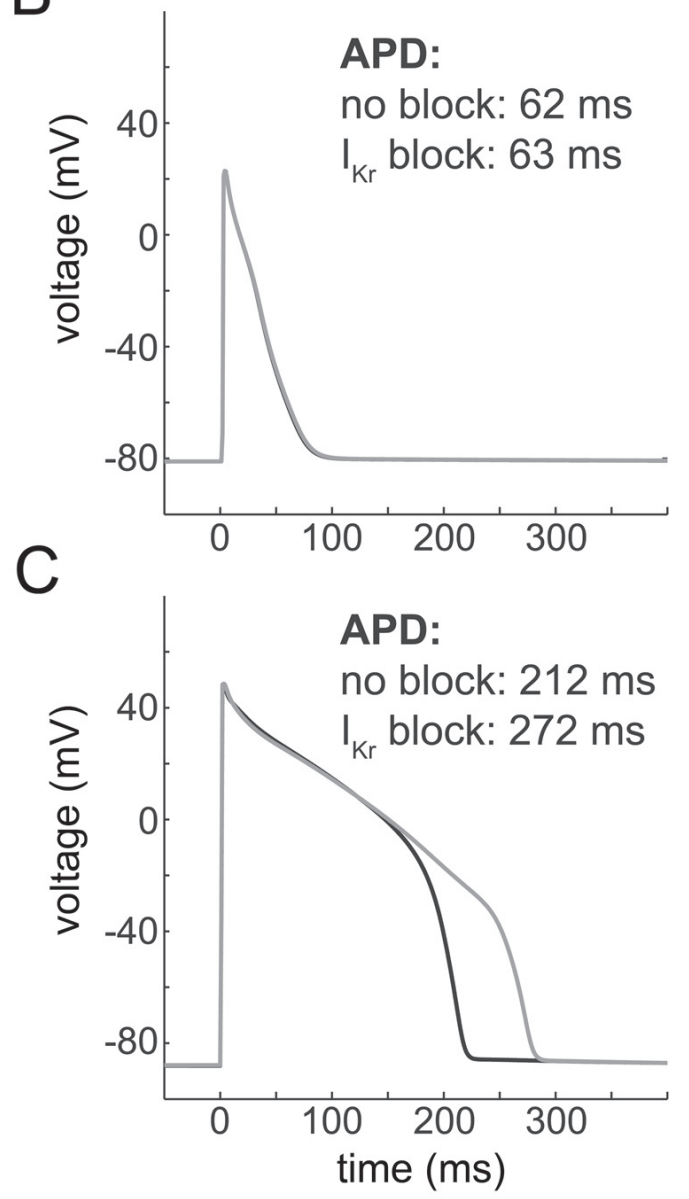

Figure 3. Cell-type transforming clamp technique. A. Scheme of the circuit; the gray area represents the target. For explanation see text. B. AP prolongation under inhibition of $I_{K r}$ cannot be observed in the mouse cardiomyocyte with the original AP configuration, i.e. AP waveform without inhibition of $I_{K r}$ and in its presence overlap. C. The change of mouse AP configuration to that typical for human provided by the cell-type transforming clamp technique enables to demonstrate AP prolongation under inhibition of $I_{K r}$. Black trace, without inhibition of $I_{K r}$; gray trace, with inhibition of $I_{K r}$; APD, action potential duration. Modified with permission from AhrensNicklas and Christini (2009).

pear. In case of the study by Ahrens-Nicklas and Christini (2009), significantly diverse calcium cycling in the mouse and human cardiomyocyte, which is not compensated by the model, is likely the most serious problem. After technical problems have been solved, the cell-type transforming clamp might become a potent tool used in a wide range of electrophysiological experiments.

\section{High-resolution scanning patch clamp (smart patch clamp)}

This modification of the patch clamp technique, developed by Gu et al. (2002), may be used in studies aimed at functional localization of ionic channels which require placing of the glass microelectrode on a distinct location of the membrane surface. The scanning ionic conductance microscopy described by Hansma et al. (1989) serves to obtain a high-resolution scan of the membrane surface. At first, the membrane surface is scanned with high resolution using a glass microelectrode with the tip radius $\sim 100 \mathrm{~nm}$ (nanopipette; Fig. $4 \mathrm{~A}$ ). The scanning is performed by moving the nanopipette over the cell at a fixed distance which is achieved by a feedback control keeping the ionic current through the nanopipette constant. Subsequently, the nanopipette is directed to the selected location of the membrane surface, for example on the T-tubule opening (Fig. 4B), and used in a common way to measure ionic currents through the channels situated at this location (the cell-attached single channel recording; Fig. 4C). 

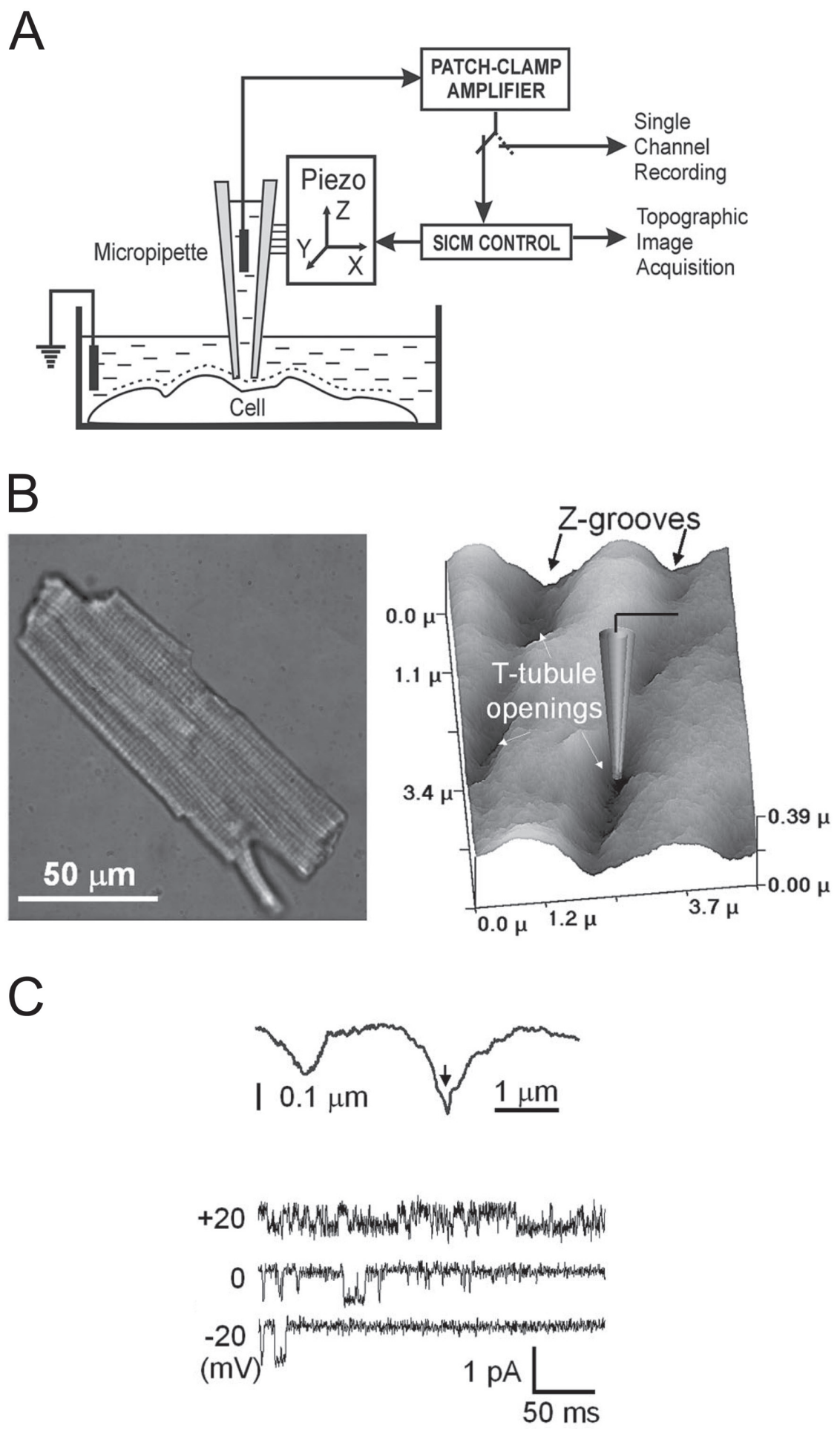

Figure 4. High-resolution scanning patch clamp. A. Schematic diagram of the setup. The glass microelectrode with the tip radius $\sim 100 \mathrm{~nm}$ (nanopipette) is mounted on the three-axis piezo actuator controlled by a computer. The ionic current flowing through the nanopipette measured by the patch clamp amplifier is used for the feedback control to keep a constant distance between the nanopipette and the scanned cell. After the scanning, computer control is used to position the nanopipette at the required location and the patch clamp measurement may start. B. Optical image of adult rat cardiomyocyte (left), and scanning ionic conductance image of the rat cardiomyocyte membrane (right). C. $\mathrm{Ba}^{2+}$ currents through the calcium channel at voltages $-20,0,+20 \mathrm{mV}$ recorded in the cell-attached mode (inset upwards: profile of the membrane, the microelectrode position is marked by the arrow). Modified with permission from Gorelik et al. (2002). 
Using this technique, $\mathrm{Gu}$ et al. (2002) found that rat cardiac L-type calcium channels are located preferentially in the T-tubules, being colocalized with chloride channels at the T-tubule openings. Density of L-type calcium channels at the T-tubule openings was estimated to $\sim 2$ channels $/ \mu \mathrm{m}^{2}$. These authors published one more paper in the same year (Gorelik et al. 2002) showing the possibility to utilize this technique in the patch clamp studies in other cell types beside cardiomyocytes (e.g. spermatozoa, neurite varicosity and fine neuronal process) and even in non-transparent samples (e.g. intact tissue preparation of the rat aorta imaging the surface of endothelial cells). According to my knowledge, no more research papers using the high-resolution patch clamp technique are available. As a matter of fact, another scientific group has recently described this technique as their own invention (Yang et al. 2010). Thus, despite promising interesting results, it seems that this technique failed to expand and to be used in the electrophysiological studies.

\section{Automated patch clamp}

Regarding the amount of acquired data, the patch clamp technique shows quite a low efficiency. Therefore, automated patch clamp systems were developed which allow measurement of hundreds to thousands of cells per day (reviewed by e.g. Mathes 2006; Clare 2010). The first systems constructed in late 1990s used the classic apposing glass microelectrodes to obtain the electrical contact with a cell (Mathes 2006). However, commercial exploitation of the automated patch clamp was particularly achieved by use of the planar patch clamp. This technology was originally developed already in 1970s (Kostyuk et al. 1975) but for the ultra-low-noise patch clamp recordings adapted in late 1990s by Klemic et al. (2002). In the planar patch clamp, the microelectrode is replaced by a glass multi-well plate (the so-called patch plate or seal chip) with 1-2 $\mu \mathrm{m}$ microscopic recording aperture placed in the middle of the bottom of every well (for figure see Clare 2010). After the suspension of cells is added into the wells, one cell per each well becomes trapped and the electrical seal is formed by applying suction. Analogically to conventional patch clamp, the patch of membrane above the aperture is then sucked away and the whole-cell recordings can be made. The common electrode is localized under the plate and the individual electrodes are merged into wells. The latest automated patch clamp systems make it possible to measure and to average the data from up to 64 cells in a single well immediately (the so-called population patch clamp; Clare 2010). By notably reducing cell-to-cell variability, the population patch clamp provides substantially more precise data (Dale et al. 2007).

In most of the currently available automated patch clamp systems, the relatively low seal resistance reaching only
$\mathrm{M} \Omega$ results in a limited voltage control. In some of them, compensation of the capacitance and series resistance is even impossible. Considering technical limitations and, thus, limited precision of recording, the obtained data are rather estimative, used for example in the early drug discovery (Choi et al. 2011; Vilums et al. 2011). However, several recent studies have proved a considerably high correlation between data obtained with the automated and conventional patch clamp (e.g. Choi et al. 2011, Scheel et al. 2011). If the remaining technical limitations are resolved in the near future, the automated patch clamp measurements may become a very effective tool for ionic current analysis.

\section{Conclusions}

This review summarizes the most important upgraded modifications of the patch clamp technique used in cardiac cellular electrophysiology. These new techniques considerably widen the possibilities of analysis of cardiac ionic channel function and their interplay, and of the contribution of particular ionic channels to AP configuration. In case of the automated patch clamp, the efficiency of this technique is many times increased. With a wider use of these techniques, we may soon expect a considerable progress in cardiac cellular electrophysiology and related fields.

Acknowledgement. The author thanks to Prof. P. Bravený for reading the manuscript and valuable comments. This work has been supported by the grant project MSM0021622402 from the Ministry of Education, Youth and Sports of the Czech Republic.

\section{References}

Ahrens-Nicklas R. C., Christini D. J. (2009): Anthropomorphizing the mouse cardiac action potential via a novel dynamic clamp method. Biophys. J. 97, 2684-2692 http://dx.doi.org/10.1016/j.bpj.2009.09.002

Banyasz T., Horvath B., Jian Z., Izu L. T., Chen-Izu Y. (2011): Sequential dissection of multiple ionic currents in single cardiac myocytes under action potential-clamp. J. Mol. Cell. Cardiol. 50, 578-581 http://dx.doi.org/10.1016/j.yjmcc.2010.12.020

Berecki G., Zegers J. G., Verkerk A. O., Bhuiyan Z. A., de Jonge B., Veldkamp M. W., Wilders R., van Ginneken A. C. (2005): HERG channel (dys)function revealed by dynamic action potential clamp technique. Biophys. J. 88, 566-578 http://dx.doi.org/10.1529/biophysj.104.047290

Berecki G., Zegers J. G., Bhuiyan Z. A., Verkerk A. O., Wilders R., van Ginneken A. C. (2006): Long-QT syndrome-related sodium channel mutations probed by the dynamic action potential clamp technique. J. Physiol. 570, 237-250 
Berecki G., Zegers J. G., Wilders R., Van Ginneken A. C. (2007): Cardiac channelopathies studied with the dynamic action potential-clamp technique. Methods. Mol. Biol. 403, 233-250 http://dx.doi.org/10.1007/978-1-59745-529-9_16

Choi K. H., Song C., Cheong C. S., Rhim H. (2011): Pharmacological studies of Cav3.1 T-type calcium channels using automated patch-clamp techniques. Gen. Physiol. Biophys. 30, 100-105 http://dx.doi.org/10.4149/gpb_2011_01_100

Clare J. J. (2010): Targeting ion channels for drug discovery. Discov. Med. 9, 253-260

Cooper P. J., Soeller C., Cannell M. B. (2010): Excitation-contraction coupling in human heart failure examined by action potential clamp in rat cardiac myocytes. J. Mol. Cell. Cardiol. 49, 911-917 http://dx.doi.org/10.1016/j.yjmcc.2010.04.012

Dale T. J., Townsend C., Hollands E.C., Trezise D.J. (2007): Population patch clamp electrophysiology: a breakthrough technology for ion channel screening. Mol. Biosyst. 3, 714-722 http://dx.doi.org/10.1039/b706152h

Danielli J. F., Davson H. (1935): A contribution to the theory of permeability of thin films. J. Cell. Comp. Physiol. 5, 495-508 http://dx.doi.org/10.1002/jcp.1030050409

Doerr T., Denger R., Doerr A., Trautwein W. (1990): Ionic currents contributing to the action potential in single ventricular myocytes of the guinea pig studied with action potential clamp. Pflügers. Arch. 416, 230-237 http://dx.doi.org/10.1007/BF00392058

Doerr T., Denger R., Trautwein W. (1989): Calcium currents in single SA nodal cells of the rabbit heart studied with action potential clamp. Pflügers. Arch. 413, 599-603 http://dx.doi.org/10.1007/BF00581808

Dong M., Sun X., Prinz A. A., Wang H. S. (2006): Effect of simulated $\mathrm{I}($ to) on guinea pig and canine ventricular action potential morphology. Am. J. Physiol. Heart. Circ. Physiol. 291, H631-637 http://dx.doi.org/10.1152/ajpheart.00084.2006

Economo M. N., Fernandez F. R., White J. A. (2010): Dynamic clamp: alteration of response properties and creation of virtual realities in neurophysiology. J. Neurosci. 30, $2407-2413$ http://dx.doi.org/10.1523/JNEUROSCI.5954-09.2010

Gorelik J., Gu Y., Spohr H. A., Shevchuk A. I., Lab M. J., Harding S. E., Edwards C. R., Whitaker M., Moss G. W., Benton D. C., Sánchez D., Darszon A., Vodyanoy I., Klenerman D., Korchev Y. E. (2002): Ion channels in small cells and subcellular structures can be studied with a smart patch-clamp system. Biophys. J. 83, 3296-3303 http://dx.doi.org/10.1016/S0006-3495(02)75330-7

Gu Y., Gorelik J., Spohr H. A., Shevchuk A., Lab M. J., Harding S. E., Vodyanoy I., Klenerman D., Korchev Y. E. (2002): High-resolution scanning patch-clamp: new insights into cell function. FASEB J. 16, 748-750

Hansma P. K., Drake B., Marti O., Gould S. A., Prater C. B. (1989): The scanning ion-conductance microscope. Science 243, 641-643 http://dx.doi.org/10.1126/science.2464851

Huelsing D. J., Spitzer K. W., Cordeiro J. M., Pollard A. E. (1998): Conduction between isolated rabbit Purkinje and ventricular myocytes coupled by a variable resistence. Am. J. Physiol. 274, H1163-1173

Huxley A. F., Hodgkin A. L. (1952): A Quantitative Description of Membrane Currentand Its Application to Conduction and Excitiation in Nerve. J. Physiol. 1, 500-544

Karmažínová M., Lacinová L. (2010): Measurement of cellular excitability by whole cell patch clamp technique. Physiol. Res. 59, $\mathrm{S} 1-7$

Klemic K. G., Klemic J. F., Reed M. A., Sigworth F. J. (2002): Micromolded PDMS planar electrode allows patch clamp electrical recordings from cells. Biosens. Bioelectron. 17, 597-604 http://dx.doi.org/10.1016/S0956-5663(02)00015-5

Kostyuk P. G., Krishtal O. A., Pidoplichko V. I. (1975): Effect of internal fluoride and phosphate on membrane currents during intracellular dialysis of nerve cells. Nature. 257, 691-693 http://dx.doi.org/10.1038/257691a0

Ling G., Gerard R. W. (1949): The normal membrane potential of frog sartorius fibers. J. Cell. Physiol. 34, 383-396 http://dx.doi.org/10.1002/jcp.1030340304

Marangoni S., Di Resta C., Rocchetti M., Barile L., Rizzetto R., Summa A., Severi S., Sommariva E., Pappone C., Ferrari M., Benedetti S., Zaza A. (2011): A Brugada syndrome mutation (p.S216L) and its modulation by p.H558R polymorphism: standard and dynamic characterization. Cardiovasc. Res. 91, 606-616 http://dx.doi.org/10.1093/cvr/cvr142

Mathes C. (2006): QPatch: the past, present and future of automated patch clamp. Expert. Opin. Ther. Targets. 10, 319-327 http://dx.doi.org/10.1517/14728222.10.2.319

Neher E., Sakmann B. (1976): Single channel currents recorded from membrane of denervated frog muscle fibers. Nature. (Lond.) 260, 799-802 http://dx.doi.org/10.1038/260799a0

Ogden D., Stanfield P. (1994): Patch clamp techniques for single channel and whole-cell recording. In: Microelectrode Techniques (2nd edition). The Company of Biologists Ltd., Cambridge

Piccolino M. (1998): Animal electricity and the birth of electrophysiology: the legacy of Luigi Galvani. Brain. Res. Bull. 46, 381-407 http://dx.doi.org/10.1016/S0361-9230(98)00026-4

Scheel O., Himmel H., Rascher-Eggstein G., Knott T. (2011): Introduction of a Modular Automated Voltage-Clamp Platform and Its Correlation with Manual Human Ether-à-go-go Related Gene Voltage-Clamp Data. Assay. Drug. Dev. Technol. 9, 600-607 http://dx.doi.org/10.1089/adt.2010.0352

Scott S. (1979): Stimulation simulations of young yet cultured beating hearts ( $\mathrm{PhD}$ thesis). State University of New York at Buffalo

Stengl M., Bartak F., Sykora R., Chvojka J., Benes J., Krouzecky A., Novak I., Sviglerova J., Kuncova J., Matejovic M. (2010): Reduced L-type calcium current in ventricular myocytes from pigs with hyperdynamic septic shock. Crit. Care. Med. 38, 579-587 http://dx.doi.org/10.1097/CCM.0b013e3181cb0f61 
Szentandrássy N., Nagy D., Ruzsnavszky F., Harmati G., Bányász T., Magyar J., Szentmiklósi A. J., Nánási P. P. (2011): Powerful technique to test selectivity of agents acting on cardiac ion channels: the action potential voltage-clamp. Curr. Med. Chem. 18, 3737-3756 http://dx.doi.org/10.2174/092986711796642418

Verkerk A. O., Veldkamp M. W., de Jonge N., Wilders R., van Ginneken A. C. (2000): Injury current modulates afterdepolarizations in single human ventricular cells. Cardiovasc. Res. 47, 124-132 http://dx.doi.org/10.1016/S0008-6363(00)00064-X

Vilums M., Overman J., Klaasse E., Scheel O., Brussee J., Ijzerman A. P. (2012): Understanding of molecular substructures that contribute to hERG $\mathrm{K}(+)$ channel blockade: synthesis and biological evaluation of E-4031 analogues. ChemMedChem. $7,107-113$ http://dx.doi.org/10.1002/cmdc.201100366

Wang Y. G., Kumar R., Wagner M. B., Wilders R., Golod D. A., Goolsby W. N., Joyner R. W. (2000): Electrical interactions between a real ventricular cell and an anisotropic two-dimensional sheet of model cells. Am. J. Physiol. Heart. Circ. Physiol. 278, H452-460

Watanabe E. I., Honjo H., Anno T., Boyett M. R., Kodama I., Toyama J. (1995): Modulation of pacemaker activity of sinoatrial node cells by electrical load imposed by an atrial cell model. Am. J. Physiol. 269, H1735-1742

Wilders R. (2006): Dynamic clamp: a powerful tool in cardiac electrophysiology. J. Physiol. 576, 349-359 http://dx.doi.org/10.1113/jphysiol.2006.115840

Yang X., Liu X., Zhang X. F., Lu H. J., Zhang Y. J. (2010): Highresolution patch-clamp technique based on feedback control of scanning ion conductance microscopy. Sheng. Li. Xue. Bao. 62, 275-283 (in Chinese)

Received: September 28, 2011

Final version accepted: November 25, 2011 